\title{
Estudio de flujos verticales de carbono y nitrógeno en ambientes acuáticos controlados en la bahía de Knebel, Dinamarca utilizando isótopos estables de nitrógeno y carbono $\left({ }^{15} \mathrm{~N}\right.$ y $\left.{ }^{13} \mathrm{C}\right)$
}

\author{
A study of the vertical flow of carbon and nitrogen in controlled aquatic environments \\ at the Knebel Bay, Denmark, with the use of the stable isotopes of nitrogen and \\ carbon $\left({ }^{15} \mathrm{~N}\right.$ y $\left.{ }^{13} \mathrm{C}\right)$
}

\section{GIOVANNI DANERI} Centro de Ciencias y Ecología Aplicada (CEA), Universidad del Mar, Carmen 446, Placeres,
Valparaíso, Chile, e-mail: gdaneri@udelmar.cl

\begin{abstract}
RESUMEN
En este estudio se utilizaron isótopos estables como trazadores para caracterizar y cuantificar el flujo vertical de carbono y nitrógeno. Los experimentos se llevaron a cabo en la bahía de Knebel, Dinamarca (56 $08^{\prime} \mathrm{N}, 10^{\circ} 11^{\prime}$ E), en dos ambientes acuáticos controlados tipo mesocosmos. La adición de nutrientes inorgánicos estimuló el afloramiento del flagelado no-tóxico Prorocentrum minimum, determinando un comportamiento similar en las mediciones de clorofila a (Clo-a), nitrógeno orgánico particulado (NOP) y carbono orgánico particulado (COP) en ambos mesocosmos. Bajo condiciones no limitantes de nutrientes inorgánicos existió una baja discriminación isotópica resultando en bajos valores de $\delta^{13} \mathrm{C}$ en el COP en suspensión y sedimentado. El desfase entre los máximos de NOP, Clo-a y COP así como la rápida asimilación del nitrato adicionado en menos de tres días y una razón $\mathrm{C} / \mathrm{N}$ variable indican que $P$. minimum posee una gran habilidad para asimilar nitrógeno inorgánico. La razón $\mathrm{C} / \mathrm{N}$ alcanzó un mínimo al inicio del experimento, para luego aumentar una vez agotado el nitrato de la columna de agua. El nitrógeno nuevo sedimentado alcanzó un 10 a $11 \%$ del total originalmente adicionado a la columna de agua en la forma de nitrato, sin que se observara una sedimentación masiva de $P$. minimum durante los días de duración de este experimento.
\end{abstract}

Palabras clave: isótopos estables, sedimentación, tramas tróficas, Prorocentrum minimum.

\begin{abstract}
Stable isotopes were used as tracers to characterize and quantify the downward flux of carbon and nitrogen. The experiments were conducted in Knebel bay, Denmark (56 $\left.08^{\circ} \mathrm{N}, 10^{\circ} 11^{\prime} \mathrm{E}\right)$, in two controlled aquatic environments (mesocosm type). The addition of inorganic nutrients to the mesocosms stimulated a bloom of the non-toxic flagellate Prorocentrum minimum. A similar pattern in the concentration of chlorophyll a (Chl-a), particulate organic nitrogen (PON) and particulate organic carbon (POC) was observed in both mesocosms. The elevated nutrient conditions resulted in low isotopic discrimination, this was reflected in low $\delta^{13} \mathrm{C}$ values of the suspended and sediment POC. The uncoupling between the peaks of PON, Chl-a and POC, as well as the rapid assimilation of the added nitrate (in less than three days) and a changing $\mathrm{C} / \mathrm{N}$ ratio indicate that $P$. minimum has a great ability to assimilate inorganic nitrogen. The $\mathrm{C} / \mathrm{N}$ ratio reached a minimum at the beginning of the experiment increasing later, once nitrate depletion was reached. The sedimentation of new nitrogen during this study was $10-11 \%$ of the total originally added to the water column. No massive sedimentation of P. minimum was observed once the nitrate added to the mesocosms was utilized.
\end{abstract}

Key words: stable isotopes, sedimentation, trophic structure, Prorocentrum minimum.

\section{INTRODUCCIÓN}

La sedimentación de la materia orgánica particulada (MOP) constituye uno de los mecanismos de exportación de carbono desde la capa ventilada de los océanos hacia el fondo marino, aportando al secuestro del anhídrido carbónico atmosférico (Shaffer 1993). Las variaciones en las tasas regionales y estacionales de sedimentación están determinadas por los procesos oceanográficos superficiales; el tipo de producción primaria (nueva, basada en $\mathrm{NO}_{3}-\mathrm{N}$ y/o regenerada, basada en $\mathrm{NH}_{4}-\mathrm{N}$ ), la estructura de la comunidad plantónica y la formación de agregaciones y feces son algunos de los factores más importantes que afectan los tiempos de retención de la materia en la columna de agua (Peinert et al. 1989). Rivkin et al. (1996) postulan que la eficiencia de retención de la materia orgánica en suspensión en las capas superficiales declina con 
eventos que interfieren con la estabilidad de las comunidades plantónicas (inyecciones de nutrientes por ejemplo). Elevadas tasas de producción primaria y bajos niveles de herbivoría han sido sugeridas como factores que favorecen rápidas tasas de sedimentación de los autótrofos una vez que los nutrientes son limitantes (Smetacek 1985, Lignell et al. 1993). A su vez, se ha postulado que el flujo vertical del carbono biogénico es mayor en tramas tróficas donde predomina la herbivoría, en donde el mayor porcentaje del carbono asimilado por los productores primarios es consumido por herbívoros, que en aquellas más complejas donde el ciclaje del carbono orgánico lo efectúan las bacterias (Roman et al. 1993). Peinert et al. (1989) informan que la magnitud del flujo vertical de materia orgánica está principalmente determinada por los niveles de producción nueva. Sin embargo, esto es solo válido cuando se promedian los flujos en grandes escalas espaciales y temporales. En situaciones específicas, el origen de la materia sedimentada puede ser producción nueva y/o regenerada y ser independiente de la estructura de la comunidad planctónica (Rivkin et al.1996).

La estimación de flujos verticales de materia orgánica formada en la columna de agua se dificulta por los siguientes problemas metodológicos: (a) la heterogeneidad del material biogénico sedimentado, (b) la existencia de aportes alóctonos y, (c) la presencia de detritus. Estos problemas se hacen más agudos en regiones costeras sujetas a descargas antropogénicas y de origen litogénico (Peinert et al. 1989).

Los métodos usados tradicionalmente para estimar la cantidad de materia orgánica que sedimenta se basan principalmente en mediciones directas de carbono orgánico particulado (COP) y/o nitrógeno orgánico particulado (NOP), utilizando el material presente en las trampas de sedimentación (Eppley 1989, Lohrenz et al. 1992, Michaels et al. 1994). También existen determinaciones en forma indirecta basadas en los cambios estacionales de los nutrientes (nitratos, fosfatos) o gases disueltos (Eppley 1989, Eppley \& Peterson 1979). Aunque cuantitativamente útiles, y pese a que se han logrado avances a través de la incorporación de análisis microscópicos de la materia orgánica particulada (MOD) sedimentada, estas técnicas no explican el origen del material orgánico sedimentado, lo que impide distinguir aportes especie específicos al flujo vertical de la materia orgánica. Lo anterior es de gran relevancia para lograr modelaciónes más efectivas de los mecanismos de transferencia del carbono desde la superficie hacia el bentos.

El análisis de la composición isotópica del COP sedimentado permite precisar su origen, debido a que los procesos biológicos alteran la razón isotópica de los compuestos biogénicos durante la fotosíntesis y, posteriormente, a través de los distintos procesos que transforman los compuestos orgánicos. El conocimiento de los procesos biológicos de discriminación isotópica es también de fundamental importancia en la interpretación de la productividad de los océanos en tiempos remotos (Raven 1993).

La composición isotópica natural de la materia orgánica en ambientes pelágicos marinos se expresa como $\delta^{13} \mathrm{C}$ en partes por mil (\%o),

$$
\delta^{13} \mathrm{C}=\left(\mathrm{R}_{\text {muestra }} / \mathrm{R}_{\text {estand }}-1\right) \cdot 1000,
$$

donde $\mathrm{R}_{\text {muestra }}={ }^{13} \mathrm{C} /{ }^{12} \mathrm{C}$ de la muestra y $\mathrm{R}_{\text {estand }}={ }^{13} \mathrm{C} /$ ${ }^{12} \mathrm{C}$ del estandard de "limestone" marino Peedee Belemnite (PDB) (Craig 1957). Como estandar primario, el PDB tiene un valor de $0 \%$, mientras que el $\delta^{13} \mathrm{C}$ del carbono inorgánico disuelto (CID) es relativamente constante en ambientes marinos (0-2,5 \%) (Craig 1957). El material biológico es más liviano (mayor cantidad de ${ }^{12} \mathrm{C}$ ) que el PDB en ambientes acuáticos debido a que durante procesos de fotosíntesis no limitados por difusión, la reducción de ${ }^{12} \mathrm{CO}_{2}$ procede a una velocidad 1,03 veces mayor que la reducción de ${ }^{13} \mathrm{CO}_{2}$ (Raven 1993) Durante la transferencia de materia orgánica a través de tramas tróficas, la razón isotópica ${ }^{12} \mathrm{C}:{ }^{13} \mathrm{C}$ sufre subsecuentes alteraciones. Incrementos sistemáticos en el $\delta^{13} \mathrm{C}$ han sido observados en cadenas tróficas acuáticas y terrestres, lo que esta probablemente asociado a la excreción selectiva y respiración de isótopos de carbón más livianos (Rau et al. 1990). Los valores de $\delta^{13} \mathrm{C}$ del plancton fluctúan entre un rango relativamente limitado: -23,8 a -19,3\% (Rounick \& Winterbourn 1986) o $-25,3$ a $-19,8 \%$ (Rau et al. 1990).

Comparaciones entre el $\delta^{13} \mathrm{C}$ de la materia orgánica suspendida y el de la materia sedimentada permiten inferir el grado de ciclaje del COP en ambientes pelágicos antes que sedimente. En sistemas con altas tasas de sedimentación de células fitoplanctónicas intactas (no consumidas por herbívoros), el $\delta^{13} \mathrm{C}$ del COP sedimentado y suspendido es similar, mientras que en sistemas donde prevalece la herbivoría y donde existe una gran producción de material fecal, el COP sedimentado es isotópicamente más pesado (3-4 \%) que el COP en suspensión (Rau et al. 1990).

Alteraciones artificiales de las proporciones isotópicas naturales de compuestos biogénicos, tales como el carbono y el nitrógeno, son también útiles en la estimación y caracterización de los flujos verticales de MOP al permitir el seguimiento de las moléculas orgánicas marcadas isotópicamente a través de tramas tróficas 
pelágicas y bentónicas. En este estudio se realizaron experimentos con isótopos estables de carbono y nitrógeno en ambientes controlados tipo mesocosmos, para caracterizar y cuantificar el flujo vertical de la MOP en condiciones de alta producción nueva, estimulada por el aumento artificial de nutrientes inorgánicos. Se estudió la hipótesis que los afloramientos de microalgas sedimentan rápidamente una vez agotados los nutrientes de la columna de agua. Bajo estas condiciones la sedimentación de células fitoplanctónicas intactas constituiría la principal vía de exportación de carbono hacia el bentos.

\section{MATERIALES Y MÉTODOS}

\section{Ubicación y características de los mesocosmos}

Los estudios se realizaron durante julio de 1992 en el fiordo de Knebel, una pequeña entrada de agua frente a la bahía de Aarhus (56 $08^{\circ}$ N, $\left.10^{\circ} 11^{\prime} \mathrm{E}\right)$, en Dinamarca. Veintidós mesocosmos fueron instalados a $200 \mathrm{~m}$ de la costa aproximadamente, en una estructura tipo balsa. El principal objetivo de estos experimentos fue estudiar el efecto de descargas antropogénicas (asociadas principalmente a cargas de nutrientes) sobre las comunidades planctónicas de mares semiabiertos. Los mesocosmos fueron construidos de mangas de plástico de una dimensión de $1,5 \mathrm{~m}$ de diámetro y aproximadamente $3 \mathrm{~m}$ de profundidad, y un volumen total aproximado de 7.0001 de agua completamente aislados. Los mesocosmos permiten la manipulación de grandes cantidades de agua por lo que los resultados de este tipo de experimentos son considerados una aproximación más representativa (con relación a manipulaciones en el laboratorio) de lo que ocurre en el medio ambiente natural. Sin embargo, su uso no está exento de complicaciones debido principalmente a la exclusión de procesos advectivos y a la acumulación de desechos metabólicos los que, en experimentos prolongados, pueden constituir una fuente de toxicidad para las comunidades estudiadas en los mesocosmos. Detalles de la construcción y puesta en marcha de estos mesocosmos se encuentran en Nybroe et al. (1992). En los experimentos del presente estudio se utilizaron dos mesocosmos, los cuales fueron enriquecidos con nutrientes inorgánicos con la intención de estimular afloramientos fitoplanctónicos. Uno de los mesocosmos fue enriquecido, al inicio del experimento, con nitrato $\left(14 \mu \mathrm{mol} \mathrm{l}^{-1}\right)$, silicato $\left(14 \mu \mathrm{mol} \mathrm{l}^{-1}\right)$ y fosfato $\left(2 \mu \mathrm{mol} \mathrm{1^{-1 }}\right)$, siendo la composición isotópica natural del nitrato alterada de modo que la proporción ${ }^{15} \mathrm{NO}_{3}:{ }^{14} \mathrm{NO}_{3}$ fue de 1:1 (peso:peso). Durante este trabajo este mesocosmo será referido como mesocosmo de abundancia isotópica artificial. Al otro mesocosmo se le adicionó, al inicio del experimento, $24 \mu \mathrm{mol} \mathrm{l}^{-1}$ de nitrato, $24 \mu \mathrm{mol} \mathrm{l}^{-1} \mathrm{de}$ silicato y $2,5 \mu \mathrm{mol} \mathrm{l}^{-1}$ de fosfato de modo que la razón isotópica natural del nitrato no fue alterada. En este trabajo, este segundo mesocosmo será referido como mesocosmo de abundancia isotópica natural. En el caso del mesocosmos de abundancia isotópica artificial la cuantificación de las tasas de sedimentación de la MOP se realizó siguiendo el isótopo estable ${ }^{15} \mathrm{~N}$ del nitrógeno orgánico particulado (NOP) en la columna de agua y en las trampas de sedimentación, la cantidad de nitrógeno nuevo $\left(\mathrm{NO}_{3}\right.$-N-basado) se estimó de acuerdo a la formula: $\mathrm{NOP} \bullet\left({ }^{15} \mathrm{~N}:{ }^{14} \mathrm{~N} / 100\right) \bullet 2$. En el caso del mesocosmo de abundancia isotópica natural la cuantificación y caracterización de las tasas de sedimentación de la MOP se hizo analizando la composición isotópica natural del COP en suspensión y el COP sedimentado en las trampas. Dificultades logísticas asociadas a la disponibilidad de mesocosmos impidieron replicar los experimentos en forma rigurosa. Sin embargo, el enriquecimiento de nutrientes inorgánicos aplicado al mesocosmo de abundancia isotópica natural y al de abundancia isotópica artificial fue similar (se adicionaron los mismos nutrientes). Las cantidades iniciales de nitrato y silicato fueron mayores en el mesocosmo de abundancia natural debido a problemas con el suministro del nitrato marcado isotópicamente $\left({ }^{15} \mathrm{~N}\right)$.

\section{Recolección de muestras}

Las muestras fueron obtenidas a intervalos crecientes de tiempo, cada 6 horas durante los primeros dos días, cada $12 \mathrm{~h}$ hasta los cuatro días y luego cada $24 \mathrm{~h}$. Tanto en el mesocosmo de abundancia isotópica artificial como en el de abundancia isotópica natural, se obtuvieron muestras superficiales discretas para el análisis de $\mathrm{NO}_{3}, \mathrm{COP}$, NOP y clorofíla a (Clo-a). El flujo vertical de la materia orgánica se estimó usando trampas de sedimentación ubicadas a 1,5 y $2,5 \mathrm{~m}$ de profundidad. Las trampas fueron construidas de cilindros de perspex de 214,8 $\mathrm{mm}$ de diámetro y fueron retiradas cada dos días. En ambos casos no se usaron preservantes ya que éstos interfieren en el análisis de la composición isotópica de las muestras.

\section{Análisis químico}

En el caso del mesocosmo de abundancia isotópica artificial el COP y NOP del material suspendido 
y sedimentado, así como la composición isotópica de las muestras enriquecidas $c{ }^{15} \mathrm{~N}$, fueron analizadas en triplicado, concentrando el MOP en filtros GF/F pre-combustionados. Los filtros con las muestras fueron tratados con $\mathrm{HCl}$ concentrado para remover el CID, secados y luego combustionados en un analizador de carbono y nitrógeno (Roboprep), instalado en línea con un espectrómetro de masa (Europa Scientific). En el caso del mesocosmo de abundancia isotópica natural la determinación del $\delta^{13} \mathrm{C}$, de la materia orgánica suspendida y sedimentada se realizó concentrando el COP en filtros GF/F precombustionados y tratados con $\mathrm{HCl}$. Las muestras fueron analizadas y procesadas con un espectrómetro de masa (VG). En ambos mesocosmos las muestras de clorofila a (Clo-a) fueron filtradas a través de filtros de fibra de vidrio (GF/C, $25 \mathrm{~mm})$. Los pigmentos fueron extraídos en acetona fría (90\%) en oscuridad por $24 \mathrm{~h}$ y posteriormente cuantificados utilizando un HPLC. Las muestras de nitrato fueron analizadas con un analizador de inyección de flujo (Tecator, Hoganas) utilizando la metodología de Grasshoff et al. (1983). El coeficiente de variación de las muestras realizadas en triplicado estuvo en el rango de 0 a $15 \%$.

\section{RESULTADOS}

\section{Mesocosmo de abundancia isotópica natural}

El incremento artificial de nutrientes en el mesocosmo de abundancia isotópica natural estimuló el crecimiento del dinoflagelado no tóxico Prorocentrum minimum, el cual se encontraba presente en la columna de agua al inicio del experimento. El contenido inicial de Clo-a aumentó de $5 \mu \mathrm{g}^{-1}$ hasta alcanzar un máximo de 33,6 $\mu \mathrm{g}^{-1}, 3,4$ días después de iniciado el experimento. El valor máximo de Cloa observado coincidió con el agotamiento del nitrato en la columna de agua (Fig. 1). El COP aumentó de un valor inicial de $73 \mu \mathrm{mol} \mathrm{l}^{-1}$ hasta un valor máximo de $367 \mu \mathrm{mol} \mathrm{l}^{-1}$, 4,8 días después del inicio del experimento. Un desfase de 1,4 días se observó entre el máximo de Clo-a y el máximo de COP (Fig. 1). La razón C/N del MOP suspendido varió de un valor inicial de 6,7 a un valor mínimo de 5,8, luego de 2,4 días de iniciado el experimento, e incrementando posteriormente en forma sostenida hasta alcanzar un valor máximo de 17,7 después de 9,4 días del inicio del experimento. Los mayores incrementos en la razón $\mathrm{C} / \mathrm{N}$ se observaron 2,8 días después de iniciado el experimento coincidiendo con el agotamiento del ni- trato y un incremento en el COP suspendido. El incremento de $P$. minimum provocó un enriquecimiento isotópico de la materia orgánica suspendida cuyo valor inicial de $\delta^{13} \mathrm{C}$ aumentó de $-17,69 \%$ hasta un rango de $-8,5$ a $-7,5 \%$ luego de 4,8 días después de iniciado el experimento (Fig. 2). La composición isotópica de la materia orgánica sedimentada no experimentó variaciones marcadas durante el período inicial del experimento; en la primera colecta, a 3,4 días de iniciado el experimento, el $\delta^{13} \mathrm{C}$ del material sedimentado medido en las trampas de 1,5 y $2,5 \mathrm{~m}$ fue $-16,63$ y $-18,55$ $\%$, respectivamente. Muestras posteriores evidenciaron un enriquecimiento isotópico del material sedimentado (Fig. 2). Sin embargo, en ningún momento el material de las trampas alcanzó un enriquecimiento isotópico similar al alcanzado por el material suspendido. Después de la primera colecta el $\delta^{13} \mathrm{C}$ del COP de la trampa más profunda fue, en promedio, un $2 \%$ mayor que el $\delta^{13} \mathrm{C}$ de la trampa de $1,5 \mathrm{~m}$ a partir del séptimo día del experimento.

\section{Mesocosmo de abundancia isotópica artificial}

Al igual que en el mesocosmo de abundancia isotópica natural, la adición de nutrientes estimu-

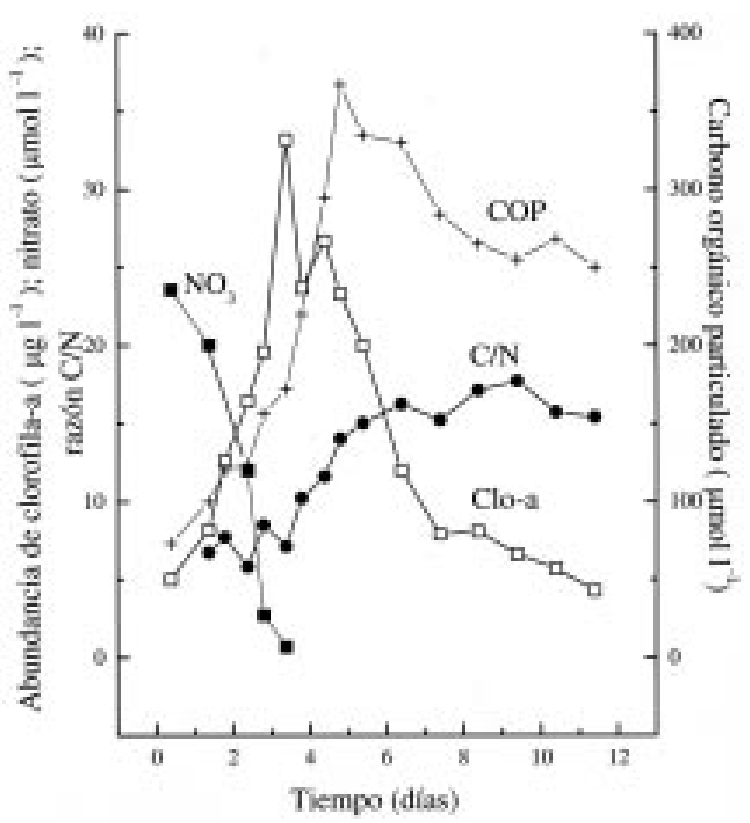

Fig. 1: Variaciones en la abundancia de clorofila a (Clo-a), nitrato $\left(\mathrm{NO}_{3}\right)$, la razón $\mathrm{C} / \mathrm{N}$ y carbono orgánico particulado (COP) en el mesocosmo de abundancia isotópica natural.

Changes in chlorophyll a (Chl-a), nitrate $\left(\mathrm{NO}_{3}\right)$, the $\mathrm{C} / \mathrm{N}$ ratio (left $\mathrm{Y}$ axis), and in the particulate organic carbon (COP) in the natural isotopic abundance mesocosm. 


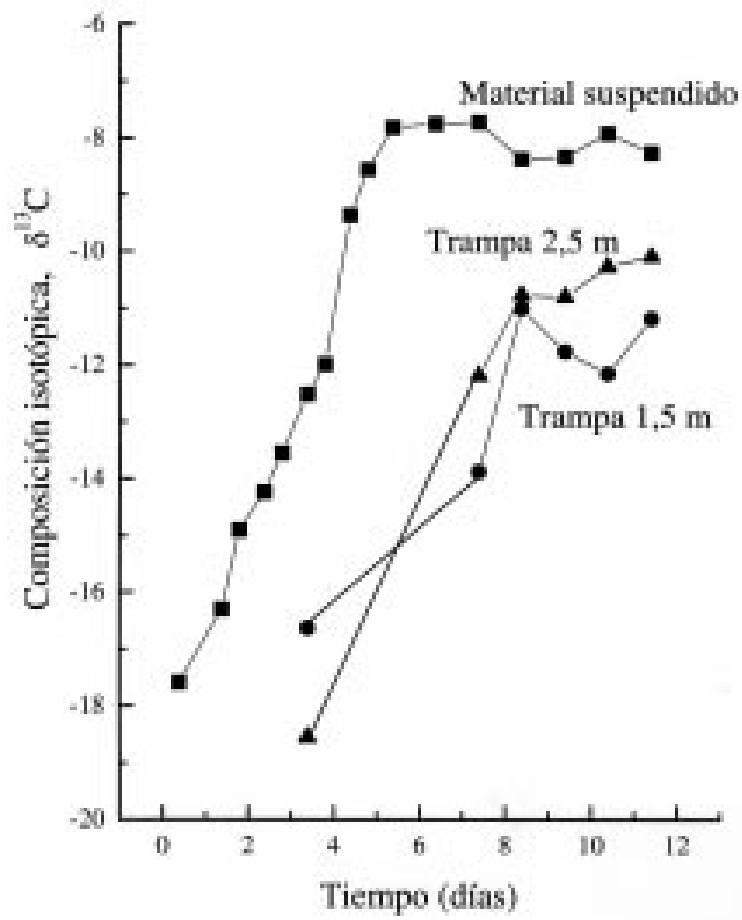

Fig. 2: Variaciones en la composición isotópica natural, estimada como $\delta^{13} \mathrm{C}$, de la materia orgánica suspendida y sedimentada en el mesocosmo de abundancia isotópica natural.

Changes in the natural isotopic composition, estimated as $\delta^{13} \mathrm{C}$, of the suspended organic matter and sediments in the natural isotopic abundance mesocosm.

ló un afloramiento de $P$. minimum, el cual agotó el nitrato inorgánico adicionado en menos de tres días (Fig. 3). El contenido de Clo-a y COP de la columna de agua aumentaron de un contenido inicial de $3,5 \mu \mathrm{g} \mathrm{l}^{-1}$ y $75 \mu \mathrm{mol} \mathrm{l}^{-1}$, respectivamente, hasta alcanzar valores máximos de $16,1 \mu \mathrm{g} \mathrm{l}^{-1} \mathrm{y}$ $194 \mu \mathrm{mol} \mathrm{l}^{-1}, 3,4$ y 3,9 días después de iniciado el experimento. En este mesocosmo se registro un desfase de 12 horas entre el máximo de Clo-a y el del COP. El C/N de la MOP en suspensión registró fluctuaciones similares a las observadas en el mesocosmo de abundancia isotópica natural, variando de un valor inicial de 7,8 a un mínimo de 6,1 después de 1,4 días de iniciado el experimento para luego aumentar y mantenerse en un rango de 16-17, luego de 5,6 días. El máximo incremento relativo de la $\mathrm{C} / \mathrm{N}$ (de 7,3 a 12,4) coincidió con el máximo de COP, 3,9 días después de iniciado el experimento (Fig. 3).

El NOP-nuevo (nitrógeno proveniente del nitrato adicionado) en suspensión y en las trampas de sedimentación experimentaron un enriquecimiento isotópico casi instantáneo producto del afloramiento de $P$. minimum. El máximo de NOPnuevo en suspensión se alcanzó 2,9 días después del inicio del experimento, $12 \mathrm{~h}$ antes del máximo de Clo-a y 24 horas antes del máximo de COP (Fig. 3 y 4). Las mayores cantidades de materia orgánica recolectada en ambas trampas se registraron durante la primera mitad (4 a 5 días) del experimento (Fig. 4). Los cálculos de balance de masa, usando en forma independiente el material sedimentado en las trampas de 1,5 y $2,5 \mathrm{~m}$, indican que durante el período de estudio el total del nitrógeno orgánico sedimentado fue de 4,28 y 4,7 mmol m${ }^{-2}$, respectivamente, lo que corresponde a un 10 y $11 \%$ del nitrógeno inorgánico agregado originalmente al mesocosmo de abundancia isotópica artificial.

\section{DISCUSIÓN}

La adición de los nutrientes inorgánicos estimularon un afloramiento del flagelado no tóxico Prorocentrum minimum en el mesocosmo de abundancia isotópica artificial y en el de abundancia isotópica natural. Esta especie dominó completamente la comunidad pelágica (U. Henrik comunicación personal) y agotó el nitrato inorgánico en un período inferior a tres días. La presencia masiva de $P$. minimum determinó un comportamien-

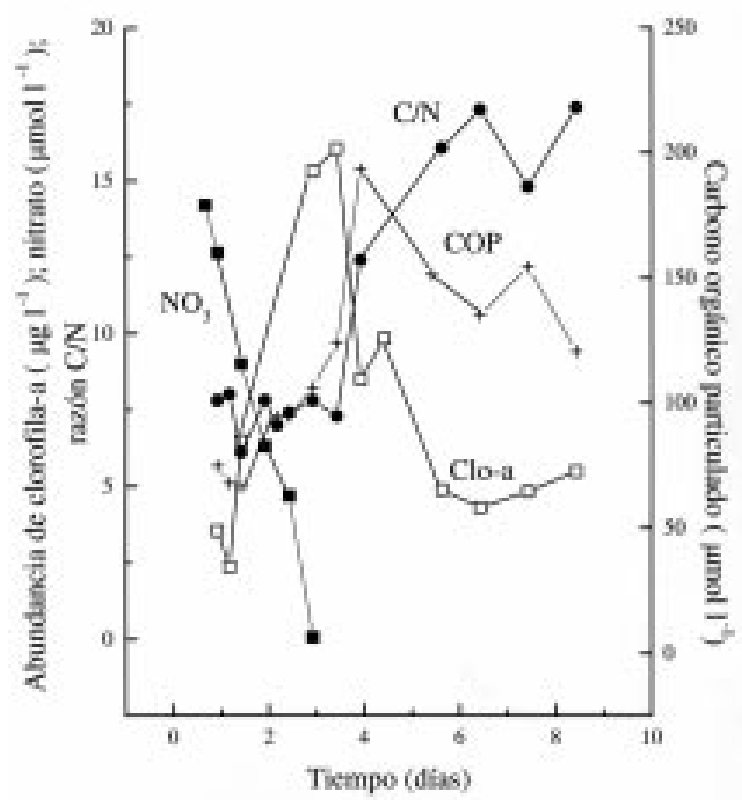

Fig. 3: Variaciones en la abundancia de clorofila a (Clo-a), nitrato $\left(\mathrm{NO}_{3}\right)$, razón $\mathrm{C} / \mathrm{N}$ y carbono orgánico particulado (COP) en el mesocosmo de abundancia isotópica artificial.

Changes in chlorophyll a (Chl-a), nitrate $\left(\mathrm{NO}_{3}\right), \mathrm{C} / \mathrm{N}$ ratio, and particulate organic carbon (COP) the artificial isotopic abundance mesocosm. 


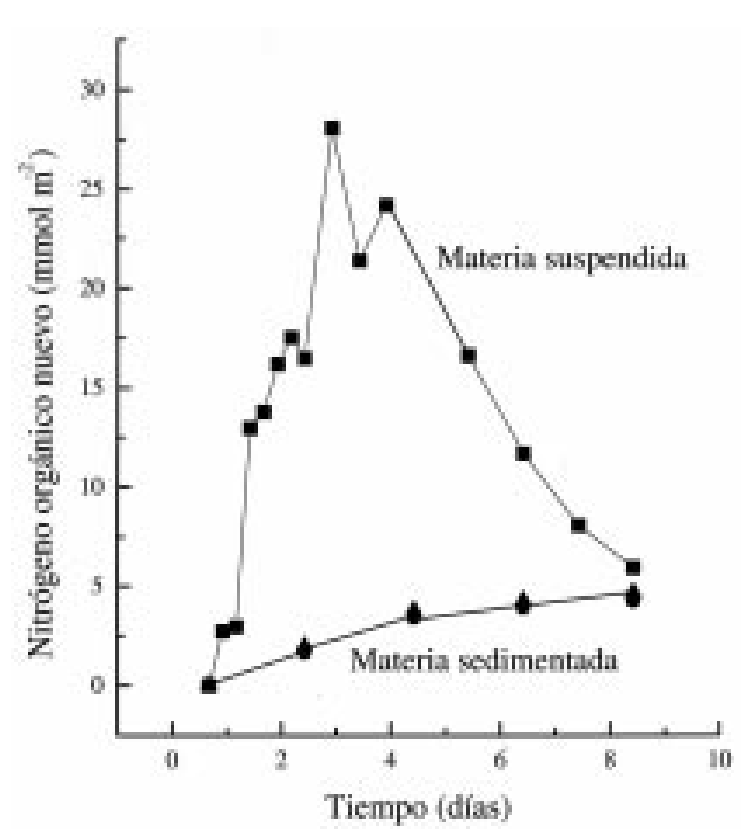

Fig. 4: Nitrógeno orgánico nuevo suspendido y sedimentado.

New organic nitrogen as suspended matter, and as sediments.

to similar de la Clo-a, el COP y la razón $\mathrm{C} / \mathrm{N}$ en ambos mesocosmos. No obstante lo anterior, en el mesocosmo de abundancia isotópica artificial los niveles de biomasa fitoplanctónica alcanzados fueron inferiores (aproximadamente la mitad en términos de Clo-a) a los niveles registrados en el mesocosmo de abundancia isotópica natural, principalmente debido a una menor adición inicial de nutrientes inorgánicos.

En el mesocosmo de abundancia isotópica natural, el "bloom" de P. minimum produjo un enriquecimiento isotópico casi instantáneo de la materia orgánica suspendida, lo que se reflejó en un aumento del $\delta^{13} \mathrm{C}$ cercano a $10 \%$. Los valores de $\delta^{13} \mathrm{C}$ para la materia orgánica en suspensión fueron más altos que los observados para sistemas abiertos (-23,85 a $-19,3 \%$, Rounick \& Winterbourn 1986; -27,4 a -26,4\%, Wada et al. 1987). Esto se debe a una baja discriminación isotópica registrada durante la síntesis de la materia orgánica, producto que en condiciones de gran abundancia de nutrientes inorgánicos, el $\mathrm{CO}_{2}$ puede limitar la producción primaria (Fry 1988, Riebesell et al. 1993, Hein \& Sand-Jensen 1997). En el mesocosmo de abundancia isotópica natural, el enriquecimiento isotópico de las trampas de sedimentación se detectó después de la segunda muestra tomada, siete días después del inicio del experimento. En ningún momento el $\delta^{13} \mathrm{C}$ del material sedimentado alcanzó valores similares al $\delta^{13} \mathrm{C}$ de la materia orgánica en suspensión. Lo anterior indicaría un desfase entre la formación de nuevo material orgánico y su posterior sedimentación, o que las tasas de sedimentación de material nuevo son mínimas en relación a la sedimentación de material en suspensión viejo, o una pérdida de compuestos isotópicamente pesados del material en suspensión previo a su sedimentación. Esta última interpretación es poco probable ya que la composición isotópica del carbono inorgánico disuelto se hizo más liviana después del alforamiento de P. minimum (Godfrey resultados no publicados). En cambio, la primera explicación se apoya en el análisis cualitativo del material de las trampas. Durante los primeros tres días el material de las trampas estuvo dominado por materia orgánica de origen indeterminado, mientras que, subsecuentemente, el material estaba dominado por células de $P$. minimum intactas. La materia orgánica contenida en la trampa más somera fue, en promedio, un $2 \%$ más pesada que la trampa más profunda, coherente con una mayor proporción de fecas de zooplancton (Godfrey resultados no publicados) las que constituyen una fuente de COP más pesada. Esta mayor proporción de feces de zooplancton se debe probablemente a que la trampa más profunda integra un mayor volumen de agua que la trampa más somera. Sin embargo, también es factible que haya existido un comportamiento migratorio del zooplancton lo que resultó en una producción de material fecal en la parte más profunda de la columna de agua. A diferencia de lo ocurrido en el mesocosmo de abundancia isotópica natural, el mesocosmo de abundancia isotópica artificial no mostró un desfase entre el enriquecimiento isotópico de la materia orgánica en suspensión y la MOP presente en las trampas, registrándose las mayores tasas de captura de NOP-nuevo los primeros 4-5 días del experimento. Lo anterior puede explicarse en términos de diferencias entre el metabolismo del carbono y del nitrógeno. En el medio ambiente marino el nitrógeno es uno de los principales factores que limitan la producción primaria por lo que algunos grupos de microalgas han desarrollado estrategias para competir en forma más exitosa por nutrientes. Una forma de competir por elementos limitantes es desacoplar la asimilación de nutrientes de la síntesis de materia orgánica, generalmente almacenándolos intracelularmente (Quay 1982). El desfase observado en los máximos de NOP-nuevo, Clo-a y COP en el mesocosmo de abundancia isotópica artificial y el desfase en los máximos de Clo-a y COP en el mesocosmo de abundancia natural, así como la rapidez con que se agotó el nitrato en ambos mesocosmos reflejan la gran habilidad de $P$. 
minimum de asimilar nitrato. Esto quedó reflejado en las fluctuaciones experimentadas por la razón $\mathrm{C} / \mathrm{N}$, la que cual inicialmente tendió a ser mínima. Por lo anterior es probable que $P$. minimum privilegie en una primera instancia la asimilación de nutrientes por sobre la síntesis de materia orgánica. Conceptualmente se puede establecer un modelo en el cual en la primera fase del desarrollo del afloramiento P. minimum asimila $\mathrm{NO}_{3}$ con una escasa síntesis de materia orgánica. Aunque en esta primera fase la sedimentación de células intactas de $P$. minimum no alteraría el $\delta^{13} \mathrm{C}$ de la materia orgánica sedimentada, tal como se observó en el mesocosmo de abundancia isotópica natural, esta induciría un enriquecimiento isotópico posterior (en términos de ${ }^{15} \mathrm{~N}$ ) en las trampas del mesocosmo de abundancia artificial. Si este modelo conceptual es correcto, la rápida asimilación de $\mathrm{NO}_{3}$ por parte de algunos grupos de algas podría significar un aporte valioso de nitrógeno al bentos, vía la sedimentación de células de fitoplancton intactas ricas en nitrógeno.

El experimento realizado en el mesocosmo enriquecido permitió una estimación del flujo total de nitrógeno hacia el bentos. Este balance indica que entre un 10 y un $11 \%$ del nitrógeno inorgánico agregado inicialmente sedimentó durante la duración del experimento, registrándose los mayores flujos al principio, sin que se observara una sedimentación masiva de $P$. minimum, como las descritas por Smetacek (1985) para un afloramiento de diatomeas. Sin embrago, los resultados concuerdan con las observaciones de Kiørboe et al. (1996), quienes al seguir el destino de un afloramiento de diatomeas y células del flagelado Phaeocystis, encontraron bajas tasas de sedimentación de células fitoplanctónicas intactas una vez agotados los nutrientes inorgánicos.

En el mesocosmo de abundancia natural el agotamiento de los nutrientes se produjo después del tercer día de iniciado el experimento, coincidiendo con el máximo de NOP-nuevo suspendido. Balances de masa indican que en el momento en que se agotó el nitrato, el NOP-nuevo suspendido y el sedimentado contenían ca. del $71 \%$ del nitrógeno originalmente adicionado (como nitrato). Este porcentaje bajó a un $26 \%$ al final del experimento, indicando un posible ciclaje del NOP-nuevo asimilado por el fitoplancton hacia otros compartimentos tales como el zooplancton herbívoro o hacia las bacterias a través de la excreción de nitrógeno orgánico disuelto (NOD). Se ha demostrado que en algunos casos la excreción de NOD puede representar hasta un $75 \%$ del nitrógeno inorgánico disuelto adicionado (Collos 1992).

Los resultados obtenidos indican que el nitrógeno es un elemento extremadamente lábil, cuyo ciclaje a través de las tramas tróficas y compartimentos reviste especial complejidad. Por lo anterior, lograr un efectivo modelaje del ciclo del nitrógeno en ambientes marinos requiere la consideración de un mayor número de procesos y reservorios de los que se consideraron en este estudio.

\section{AGRADECIMIENTOS}

Se agradece a los Drs. Carmen Morales y Humberto González por una revisión crítica de este manuscrito cuya escritura fue apoyada por el Programa Fondap-Humboldt. Se agradecen también los comentarios de un revisor anónimo y las sugerencias editoriales que ayudaron a mejorar el presente trabajo.

\section{LITERATURA CITADA}

CRAIG H (1957) Isotopic standards for carbon and oxygen and correction factors for mass-spectrometric analysis of carbon dioxide. Geochimica Cosmochimica Acta 12: $133-149$.

COLLOS Y (1992) Nitrogen budgets and dissolved organic matter cycling. Marine Ecology Progress Series 90: 201-206.

EPPLEY RW \& BJ PETERSON (1979) Particulate organic matter flux and planctonic new production in the deep ocean. Nature 282: 677-680.

EPPLEY RW (1989) New production: history, methods, problems. En: Berger WH, VS Smetacek \& G Wefer (eds) Productivity of the ocean: present and past: 85 97. John Wiley \& Sons Limited, Chichester, New York.

FRY B (1988) Food web structure on Georges Bank from stable $\mathrm{C}, \mathrm{N}$ and $\mathrm{S}$ isotopic composition. Limnology Oceangraphy 33: 1182-1190.

GRASSHOFF K, M ERHARDT \& K KREMLING (1983) Methods of seawater analysis. Springer-Verlag, Basel, Switzerland. 419 pp.

KIÅRBOE T, JLS HANSEN, AL ALLDREDGE, GA JACKSON, U PASSOW, HG DAM, DT DRAPEAU, A WAITE \& CM GARCIA (1996) Sedimentation of phytoplankton during a diatom bloom: rates and mechanisms. Journal of Marine Research 54: 11231148.

LOHRENZ SE, GA KNAUVER, VL ASPER, M TUEL, AF MICHAELS \& AH KNAP (1992) Seasonal variability in primary production and particle flux in north western Sargasso Sea: U.S. JGOFS Bermuda Atlantic time-series study. Deep Sea Research 39: 1373-1391.

LIGNELL R, AS HEISKANEN, H KUOSA, K GUNDERSEN, P KUUPPO-LEINIKKI, R PAJUNIEMI \& A UITTO (1993) Fate of a phytoplankton spring bloom: sedimentation and carbon flow in the planktonic food web in the northern Baltic. Marine Ecology Progress Series 94: 239-252. 
MICHAELS AF, AH KNAP, RL DOW, K GUNDERSEN, RJ JOHNSON, J SORENSEN, A CLOSE, GA KNAUER, SE LOHRENZ, VA ASPER, M TUEL \& R BIDIGARE (1994) Seasonal patterns of ocean biogeochemistry at the U.S. JGOFS Bermuda Atlantic time-series study site. Deep Sea Research 41: 10131038.

NYBROE O, K CHRISTOFFERSEN \& B RIEMANN (1992) Survival of Bacillus licheniformis in seawater model ecosystem. Applied Environtal Microbiology 58: 252-259.

HEIN M \& K SAND-JENSEN (1997) Kinetics of inorganic carbon use among aquatic photosynthetic organisms. Nature 388: 526-527.

PEINERT R, B VON BODUNGEN \& VS SMETACEK (1989) Food web structure and loss rate. En: Berger WH, VS Smetacek \& G Wefer (eds) Productivity of the ocean: present and past: 35-48. John Wiley \& Sons Limited, Chichester, New York.

QUAY D (1982) Effect of growth conditions on accumulation of internal nitrate, ammonium, amino acids, and protein in three marine diatoms. Journal Experimental Marine Biology Ecology 61: 243-264.

RAU GH, J-L TETSSIE, F RASSOULZADEGAN \& SW FOWLER $(1990){ }^{13} \mathrm{C} /{ }^{12} \mathrm{C}$ and ${ }^{15} \mathrm{~N} /{ }^{14} \mathrm{~N}$ variations among size fractionated marine particles: implications for their origin and trophic relationship. Marine Ecology Progress Series 59: 33-38.

RAVEN JA (1993) The potential of molecular biological and stable isotopic ideas and techniques for field measurements of marine primary productivity. ICES Marine Sciences Symposium 197: 37-41.
RIEBESELL U, D WOLF-GLADROW \& V SMETACEK (1993) Carbon dioxide limitation of marine phytoplancton growth rates. Nature 361: 249-251.

RIVKIN RB, L LEGENDRE, D DEIBEL, JE TREMBLAY, B KLEIN, K CROCKER, S ROY, N SILVERBERG, C LOVEJOY, F MESPLE, N ROMERO, MR ANDERSON, P MATTHEWS, C SAVENKOFF, A VEZINA, JC THERRIAULLT, J WESSON, C BERUBE \& RG INGRAM (1996) Vertical fluxes of biogenic carbon in oceans: is there food web control? Science 272: 1163-1166.

ROMAN M, H DAM, A GANZENS \& J NAPP (1993) Zooplancton biomass and grazing at the JGOFS Sargasso Sea time series station. Deep Sea Research 40: 883-901.

ROUNICK JS \& MJ WINTERBOURN (1986) Stable carbon isotopes and carbon flow in ecosystems. BioScience 36: 171-177.

SHAFFER G (1993) Effects of the marine biota on global carbon cycling. En: Heimann M (ed) The global carbon cycle: 431-455. Springer-Verlag, Berlin, Germany.

SMETACEK VS (1985) Role of sinking in diatom lifehistory cycles: ecological, evolutionary and geological significance. Marine Biology 84: 238-251.

WADA E M, Y TERAZAKI \& T KABAYA (1987) ${ }^{15} \mathrm{~N}$ and ${ }^{13} \mathrm{C}$ in the Antarctic Ocean with emphasis on the biogeochemical structure of food web. Deep Sea Research 34: 829-841. 